Communications in Physics, Vol.21, No. 4 (2011), pp. 341-351

\title{
DIFFUSION CHARACTERISTICS OF PARTICLES ON ENERGETICALLY DISORDERED LATTICES
}

\author{
TRINH VAN MUNG, PHAM KHAC HUNG, AND PHAM NGOC NGUYEN \\ Hanoi University of Science and Technology
}

\begin{abstract}
We present here the result of MC simulation for diffusion of particles in the lattices with both types of disorders. We have calculated the correlation factor $F$, the mean time between two subsequent jumps and the coverage dependence of diffusion coefficient. It is shown that the character of particle migration depends on the type of energetic disorder. The lattice inhomogeneity produces specific peculiarities on the coverage dependence of diffusion coefficient. For the lattice of site disorder and two-level distribution we observe an anomalous behavior of diffusion at low concentration of low energy level and at low temperature.
\end{abstract}

\section{INTRODUCTION}

Diffusion and conductivity of disordered system have received wide attention for last two decades [1-5]. It was revealed that for strong disordered system like amorphous alloys atomic transport was influenced essentially by energetic disorder and little by geometrical disorder [6-7]. Hence one can study this topic using the disordered lattice instead, where the sites are arranged in regular lattice, but site or transition (saddle point) energies are adopted randomly in accordance to given distribution [8-12]. Furthermore, the data derived from disordered lattice has been compared to crystal lattice which is denoted to the system where sites are arranged in regular lattice as disordered lattice, but site and transition energies are kept constant. For the simple case when only one particle moves in disordered lattice two specific effects were found [13-14]. First one relates to reduce the mean square displacement of particle, i.e. $\left\langle x_{n}\right\rangle^{2} \sim F n a^{2}$. Here $a, n$ is the length and number of jump; $F$ is correlation factor. In the case of crystal lattice factor $F$ is equal to 1.0, but it approached to very small value at low temperature for disordered lattice. Second effect gives rise to that the time between two consecutive jumps is much less than averaged particle resident time at site. As a result, the temperature dependence of diffusion coefficient appears to be non- or Arrhenius type depending on the strength of site or transition disorder. The situation becomes more complex when consider many particles taking part in diffusion. In this case the particles prevent each other freely move through the system (blocking effect). Moreover, the particle-particle interaction also affects on the diffusion behavior [15-17]. In actual systems, it was found the impurity concentration dependence of diffusion coefficient [18]. For example, the activation energy and diffusion coefficient $D$ for hydrogen in $\mathrm{Zr}_{69.5} \mathrm{Cu}_{12} \mathrm{Ni}_{11} \mathrm{Al}_{7.5}$ metallic glass noticeably change in the intermediate concentration regime $0.2<H / M<0.9$ [18]. Here $H / M$ is hydrogen-tometal ratio. In the low concentration regime $H / M<0.2$ insignificant maximum appears 
on the dependence of $D$ on $H / M$. Besides the hydrogen diffusivity follows the classical over-barrier-hopping mechanism. The theoretical treatment of diffusion in such systems is also very complicated. A number of models have been proposed and well described the specific features of diffusion in disordered systems (see ref. [19-22]). However, very little systematic works concerning the simulation of many-particle diffusion in the system with both kinds of energetic disorders were found in the literature yet, although the blocking effect is noticeable from both theoretical and experimental views. This motivated us to carry out a study focusing on this topic.

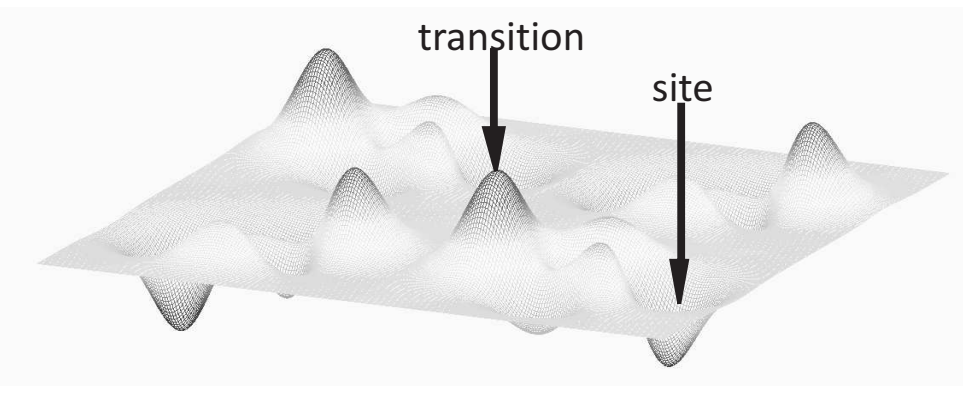

Fig. 1. Schematic representation distribution of site $\varepsilon_{i}$ and saddle-point energies $\varepsilon_{i j}$ in the two-dimensional lattice

In the present work we probe the diffusion of particles in two-dimensional lattice with site and transition disorders using Monte-Carlo (MC) method (see Fig. 1). The particleparticle interaction play its own role which is interesting and intensively investigated $[17$, 23], but they have no essential relation to the role of energetic disorder and event shadows its influence. Hence, the lattices with non-interacting particles are employed here, and both aspects: energetic disorder and blocking effect, have been studied in two separate systems: the lattice A where the transition energies are constant but site energies have randomly distributed, and lattice $\mathrm{B}$ that conversely, the transition energies are adopt randomly and site energies are kept constant.

\section{CALCULATION PROCEDURE}

Here we consider a square lattice consisting of $50 \times 50$ sites with periodic boundary conditions. The lattice coordination number is equal to 4 and the lattice constant is kept unchanged during the simulation. The energy of each site or the transition energy between two nearest neighboring sites is assigned in a random way from two-level distribution, i.e. the energy is adopt to given value $\varepsilon_{1}$ or $\varepsilon_{2}$. In the case of lattice $A$ the energies $\varepsilon_{1}, \varepsilon_{2}$ are equal to -1 and 0 , respectively; and for lattice B the corresponding values are 0 and 1 . By this way both lattices have the same energy distribution of barriers. We introduce the concentration $\alpha$ of energy level $\varepsilon_{1}, \varepsilon_{2}$ for the lattice A, B, respectively and the particle coverage $\theta=N_{p} / N$; here $N$ is number of sites in the lattice; $N_{p}$ is the number of particles. As the site and transition energies are set up for the lattice, the probability of particle's jump from $i^{\text {th }}$ to $j^{\text {th }}$ neighboring site and the averaged particle resident time at $i^{\text {th }}$ site is 
defined as

$$
\begin{array}{r}
p_{i j}=\frac{\exp \left(-\varepsilon_{i j} \beta\right)}{\sum_{l}^{4} \exp \left(-\varepsilon_{i l} \beta\right)}, \\
\tau_{i}=\frac{4 \tau_{0} \exp \left(-\varepsilon_{i} \beta\right)}{\sum_{l}^{4} \exp \left(-\varepsilon_{i l} \beta\right)} .
\end{array}
$$

where $\varepsilon_{i}$ and $\varepsilon_{i j}$ are the site and transition energies; $\tau_{0}$ is frequency period. $\beta=1 / k T ; k$ is Boltzmann constant and $T$ is temperature; the sum in Eq. (1) and Eq. (2) is taken over all transition energies of site $i$.

After construction of the lattice described above the sites are filled with a number of particles $N_{p}$ by randomly choosing their coordinate and by avoiding double occupancy. Initially a list of points (LSP) $t_{11}, t_{21}, \ldots t_{N 1}$ is determined.

Here $t_{i 1}$ is the moment that $i^{\text {th }}$ particle jumps. Because of the jump which carries the particle out of site $i$ represents the Poisson process with averaged residence time $\tau_{i}$, hence the time $t_{i 1}$ is defined as

$$
t_{i 1}=-\tau_{i} \ln R,
$$

where $R$ is random number in interval $[0,1]$. Then we select a particle $j$ that has minimum $t_{j 1}$ and then move it into neighboring site if this site is empty. The neighboring site is randomly chosen in accordance to probabilities in equation (1). In the case that the neighboring site is occupied, the particle $j$ remains on the current site, but the time of its next jump is recalculated. In the next step $n+1$ LSP is updated as

$$
t_{j n+1}=t_{j n}-\tau_{j} \ln R \text { and } t_{i n+1}=t_{i n} \text { if } i \neq j, \quad n=1,2 \ldots
$$

Such, the diffusion process is simulated. For each MC run the elapsed time $t_{n} / t_{1}^{*}$ and mean square displacement $\left\langle x_{n}^{2} / a^{2}\right\rangle$ have been calculated. Here $a$ is spacing between nearest neighboring sites; $t_{n}$ is the time realizing $n$ particle jumps. The parameter with subscript ${ }^{*}$ is denoted for crystal lattice. The data points plotted as $<x_{n}^{2} / a^{2}>$ versus $n$ and $t_{n} / t_{1}^{*}$ should be on a line with slope of correlation factor $F$ and diffusion coefficient $D / D^{*}$, respectively. In order to attain a good statistic all quantities is obtained by averaging over $10^{6}-10^{7} \mathrm{MC}$ samplings.

\section{RESULT AND DISCUSSION}

Let us consider the migration of one particle in the lattice A and B. In accordance to Eq. (1) for the lattice A the probability of particle jump from present site to neighboring site is equal to $1 / 4$. Hence the migration of particle in this lattice likes a random walk through crystal lattice, e.g. the mean square displacement follows an classical relation $<x_{n}^{2}>=n a^{2}$.

In addition, the mean time between two subsequent jumps $\tau_{\text {jump }}$ is approached to the time $\tau_{\text {res }}$ obtained by averaging the time $\tau_{i}$ (see Eq. (2)) over all sites in the lattice

$$
\tau_{\text {res }}=\frac{\sum_{i}^{N} \tau_{i}}{N} .
$$




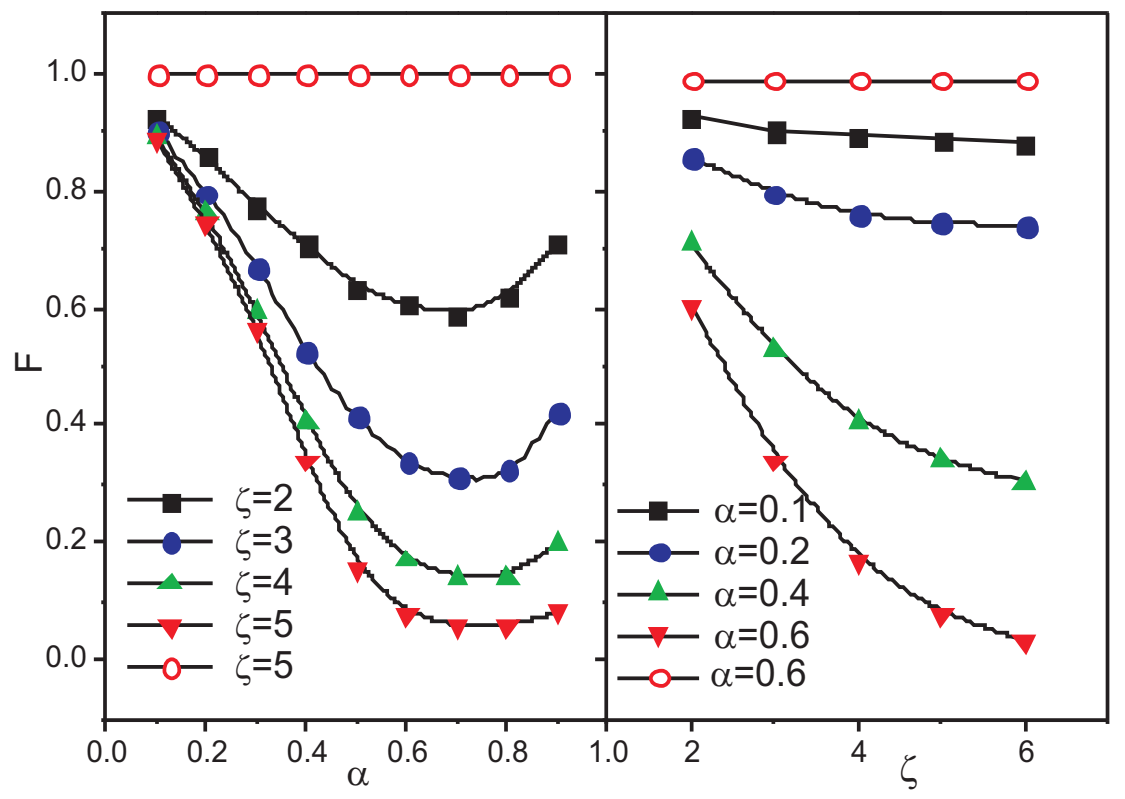

Fig. 2. The correlation factor $F$ for the case of one particle; filled symbol represents the data for lattice A and unfilled symbol for lattice B

In the case of lattice B the particle prefers to move along a path enriched by low transition energies. This results in that one obtains $\left\langle x_{n}^{2}\right\rangle=F n a^{2}$ instead. The correlation factor $F$ depends on the temperature and attains very small value at low temperature. On the other hand the time $\tau_{\text {jump }}$ is significant less than time $\tau_{r e s}$. The diffusion coefficient for disordered lattice can be given as [14]

$$
\frac{D}{D^{*}}=F \frac{\tau^{*}}{\tau_{j u m p}}
$$

Table 1. The diffusion quantities

\begin{tabular}{cccccccccc}
\hline \multirow{2}{*}{$\alpha$} & $\zeta$ & \multicolumn{3}{c}{ Lattice A } & \multicolumn{5}{c}{ Lattice B } \\
\cline { 2 - 9 } & & $\tau_{\text {jump }} / \tau^{*}$ & $\tau_{\text {ejump }} / \tau^{*}$ & $D / D^{*}$ & $F$ & $\tau_{\text {jump }} / \tau^{*}$ & $\tau_{\text {ejump }} / \tau^{*}$ & $\tau_{\text {res }} / \tau^{*}$ & $D / D^{*}$ \\
\hline 0.2 & 2 & 2.28 & 2.28 & 0.4345 & 0.8575 & 1.21 & 1.21 & 1.28 & 0.7090 \\
0.2 & 3 & 4.82 & 4.82 & 0.2055 & 0.7938 & 1.23 & 1.23 & 1.35 & 0.6427 \\
0.2 & 4 & 11.72 & 11.72 & 0.0845 & 0.7589 & 1.24 & 1.24 & 1.42 & 0.6097 \\
0.2 & 5 & 30.48 & 30.48 & 0.0325 & 0.7444 & 1.25 & 1.25 & 1.54 & 0.5963 \\
0.4 & 2 & 3.55 & 3.56 & 0.2788 & 0.7070 & 1.53 & 1.53 & 1.79 & 0.4625 \\
0.4 & 3 & 8.63 & 8.63 & 0.1148 & 0.5268 & 1.61 & 1.61 & 2.22 & 0.3264 \\
0.4 & 4 & 22.43 & 22.44 & 0.0442 & 0.4041 & 1.65 & 1.65 & 3.02 & 0.2453 \\
0.4 & 5 & 59.95 & 59.97 & 0.0165 & 0.3387 & 1.66 & 1.66 & 5.04 & 0.2042 \\
\hline
\end{tabular}


For lattice $\mathrm{A}, F=1$ and $\tau_{\text {jump }}=\tau_{\text {res }}$, e.g. the site disorder affects on the diffusion coefficient only through the time $\tau_{\text {res }}$. In converse the transition disorder decreases both the correlation factor and time $\tau_{\text {jump }}$ with decreasing the temperature.

\section{III.1. Diffusion of single particle}

As mention above, the migration for the case when there is only one particle, likes a random walk without blocking effect. This case is used to compare the diffusion in the lattice with many particles and whereby allows clarifying the blocking effect. A typical result is shown in figures 2 and 3 . Here $\zeta=\left(\varepsilon_{2}-\varepsilon_{1}\right) \beta$. The case of having site disorder gives an expected correlation factor of 1.0 which is independent on temperature.

The transition disorder gives rise to monotony decreasing the factor $F$ with decreasing the temperature. This is caused by that at low temperature the particle performs many forward and backward jumps located inside a small region of the lattice. The factor $F$ is also sensitive to the concentration $\alpha$ which is denoted to the concentration of energy level $\varepsilon_{1}$ and $\varepsilon_{2}$ for the lattice $A$ and $B$, respectively. As shown in Fig. 2 there is a pronounced minimum near $\alpha=0.75$ which is independent on temperature.

The time $\tau_{\text {jump }}$ exponentially increases with temperature in the case of lattice A, whereas this time slightly changes for lattice B and at regime of low concentration $\alpha$ the time $\tau_{\text {jump }}$ is almost unchanged with temperature. This can be explained by that at low concentration $\alpha$ the particle bypasses the high barrier surmounting only over low transition energy.

Consider the diffusion of single particle for enough long time. The time which particle spent at $i^{\text {th }}$ site can be approximated by

$$
t_{i}=t_{n} \frac{\exp \left(-\varepsilon_{i} \beta\right)}{\sum_{j}^{N} \exp \left(-\varepsilon_{j} \beta\right)} .
$$

Using equations (2) and (7) the number of visiting times of particle for $i^{\text {th }}$ site is

$$
n_{i}=\frac{t_{i}}{\tau_{i}}=t_{n} \frac{\sum_{l}^{4} \exp \left(-\varepsilon_{i l} \beta\right)}{4 \tau_{0} \sum_{j}^{N} \exp \left(-\varepsilon_{j} \beta\right)} .
$$

The averaged time between two subsequent jumps can be written as

$$
\tau_{\text {ejump }}=\frac{t_{n}}{\sum_{i}^{N} n_{i}}=\frac{4 \tau_{0} \sum_{j}^{N} \exp \left(-\varepsilon_{i l} \beta\right)}{4 \tau_{0} \sum_{j}^{N} \exp \left(-\varepsilon_{j} \beta\right)} .
$$

For the case of $\alpha=0.2$ and 0.4 the result obtained by MC calculation is presented in Table 1. It is worth to note that the time $\tau_{\text {jump }}$ is close to $\tau_{\text {ejump }}$ and diffusion coefficient for lattice $\mathrm{A}$ is significant less than one for lattice $\mathrm{B}$ at low temperature, although both lattices 


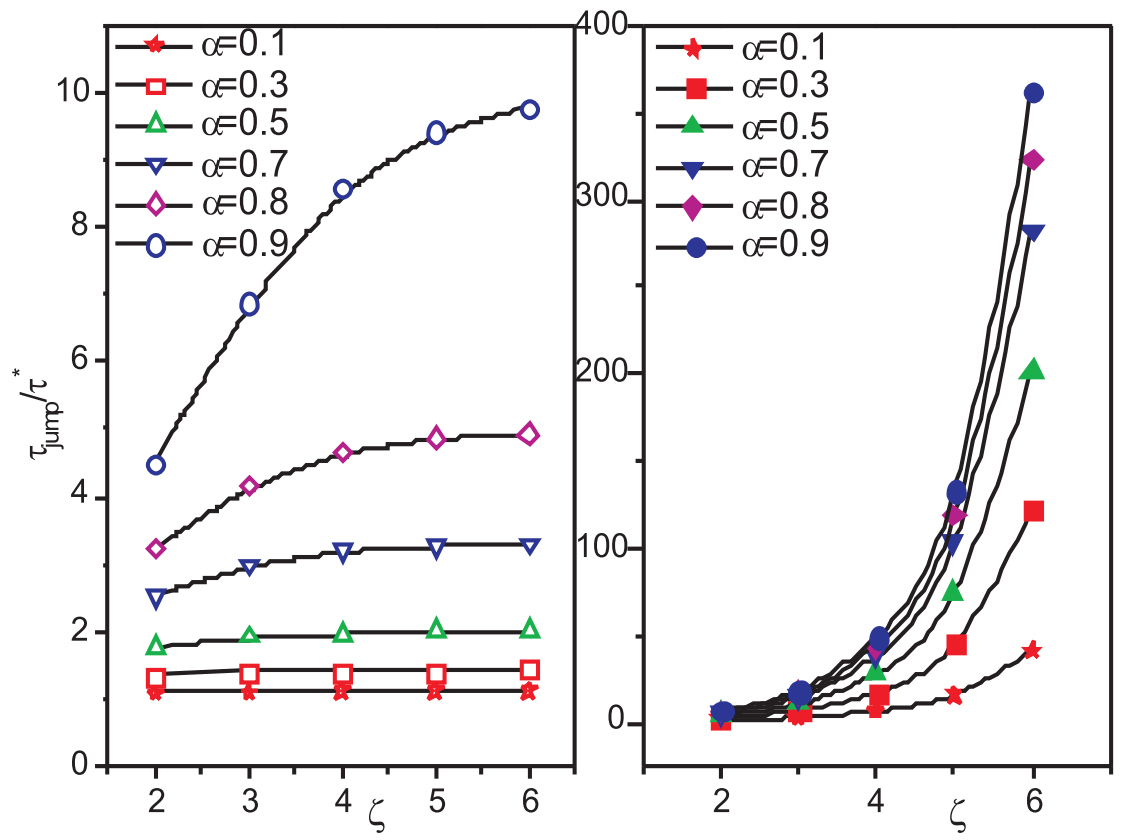

Fig. 3. The averaged time between two consequent jumps for the case of one particle; Unfilled symbol represents the data for lattice A and filled symbol for lattice B

have the same barrier distribution. Using equations (6) and (9) the diffusion coefficient for lattice A can be defined by

$$
\frac{D}{D^{*}}=\frac{\tau^{*}}{\tau_{0}}\left[\alpha \exp \left(-\varepsilon_{1} \beta\right)+(1-\alpha) \exp \left(-\varepsilon_{2} \beta\right)\right]
$$

In Fig. 4 the Arrhenius plots for several cases are shown. For both types of disorder at regime of high concentrations $\alpha$ the straight lines are observed. However, in the interval $\alpha$ from 0.1 to 0.4 it is clearly seen the deviation from Arrehenius behavior. The origin consists in that at low temperature the contribution of high transition or site energies is essential, e.g. the particle surmounts only over low barriers (lattice B) or the time $\tau_{\text {jump }}$ is defined mainly by the site energies $\varepsilon_{1}=-1$ (lattice $A$ ).

\section{III.2. Diffusion of many particles}

Figure 5 represents the Arrhenius plots for the lattice with the particle coverage $\theta=0.02$. The shape of graphs is very similar to one shown in Fig. 4 for the case of one particle. It means that blocking effect almost does not influence on the diffusion behavior in the considered lattices. To elucidate the strength of blocking effect we examine the dependence of ratio $D_{\theta} / D_{1}$ as a function of the coverage $\theta$. As shown in Fig. 6 for the lattice $\mathrm{B}$ the ratio $D_{\theta} / D_{1}$ monotony decreases in likely-linear manner with increasing the particle coverage. Whereas in the case of lattice $\mathrm{A}$ it shows an anomalous behavior: at the concentration $\alpha=0.05$ and temperature $\zeta=5$ the ratio $D_{\theta} / D_{1}$ monotony increases with the particle coverage. As the concentration $\alpha$ and temperature increases, this effect 


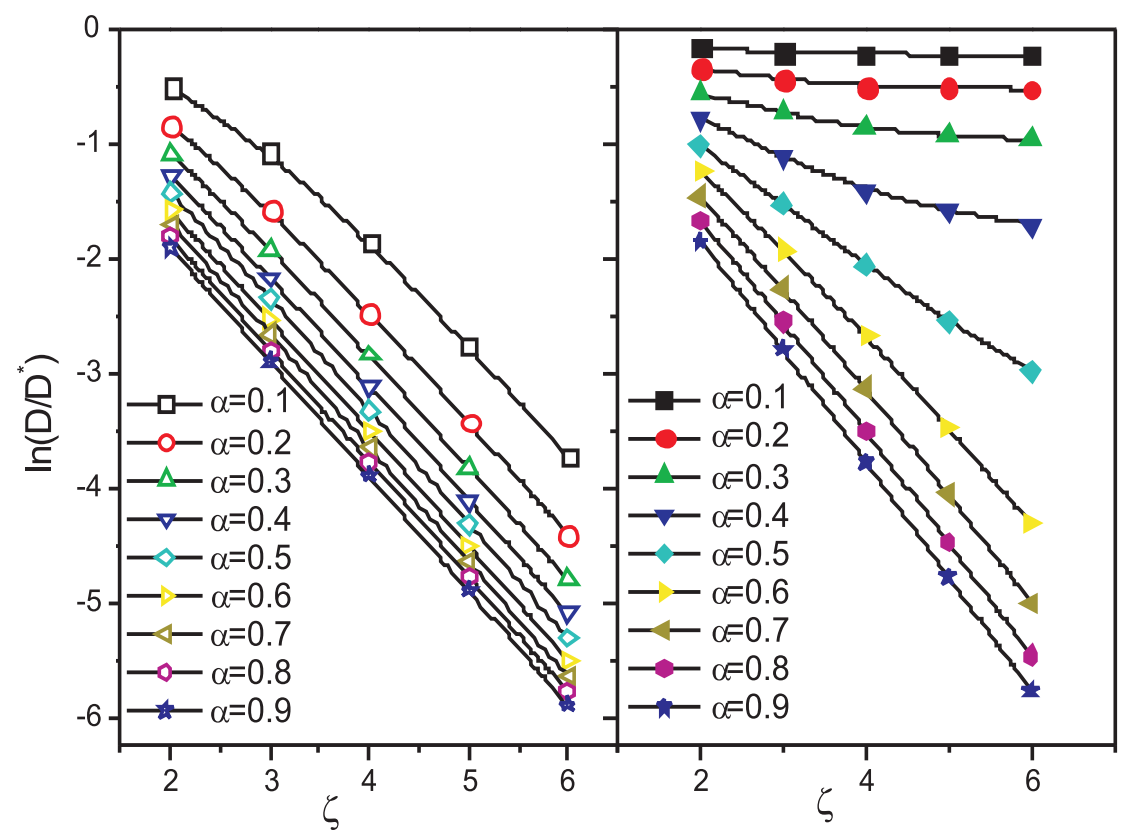

Fig. 4. The Arrhenius plot for the case of one particle: lattice A (lelft) and lattice B (right).

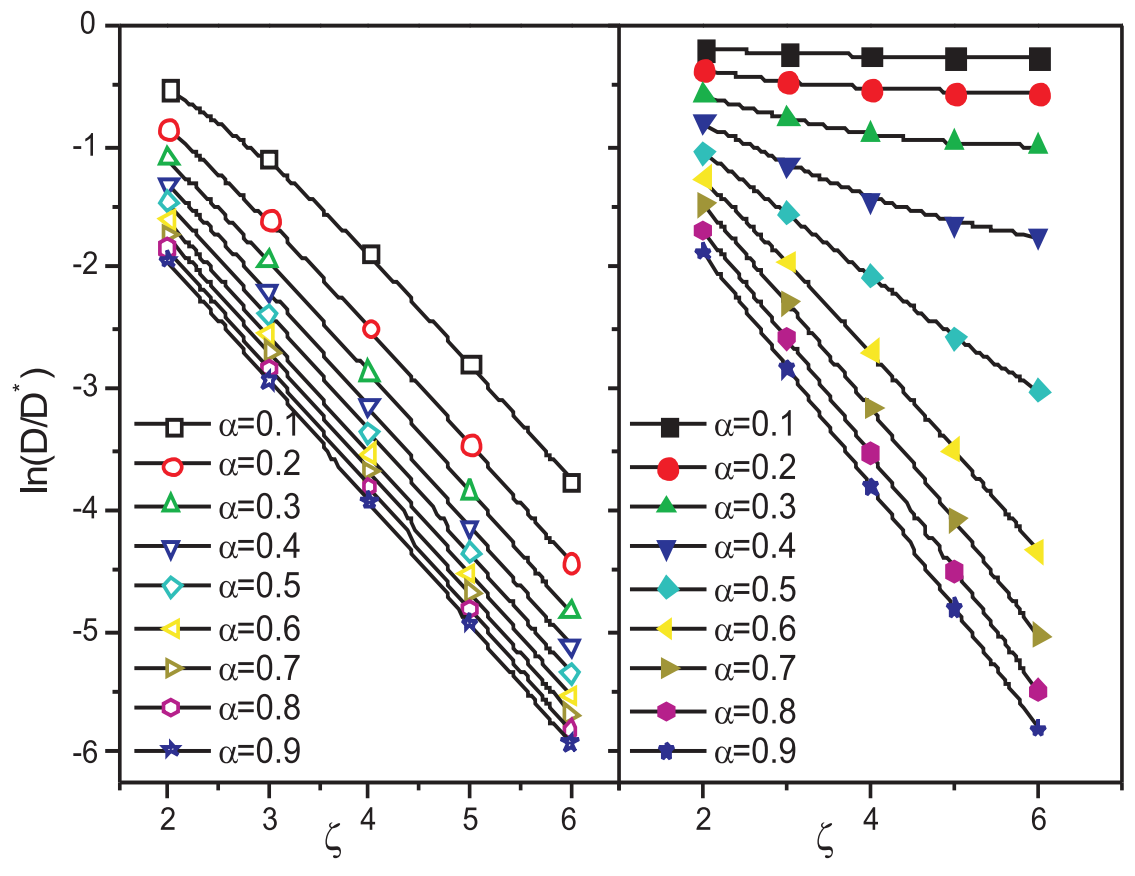

Fig. 5. The Arrhenius plot for the case with $\theta=0.02$ : lattice A (left) and lattice B (right). 
disappears. The reason of this effect will be discussed latter. In the magnitude the ratio $D_{\theta} / D_{1}$ changes from 1 to 0.65 and 1.35 to 0.68 for the lattice $\mathrm{B}$ and A, respectively.
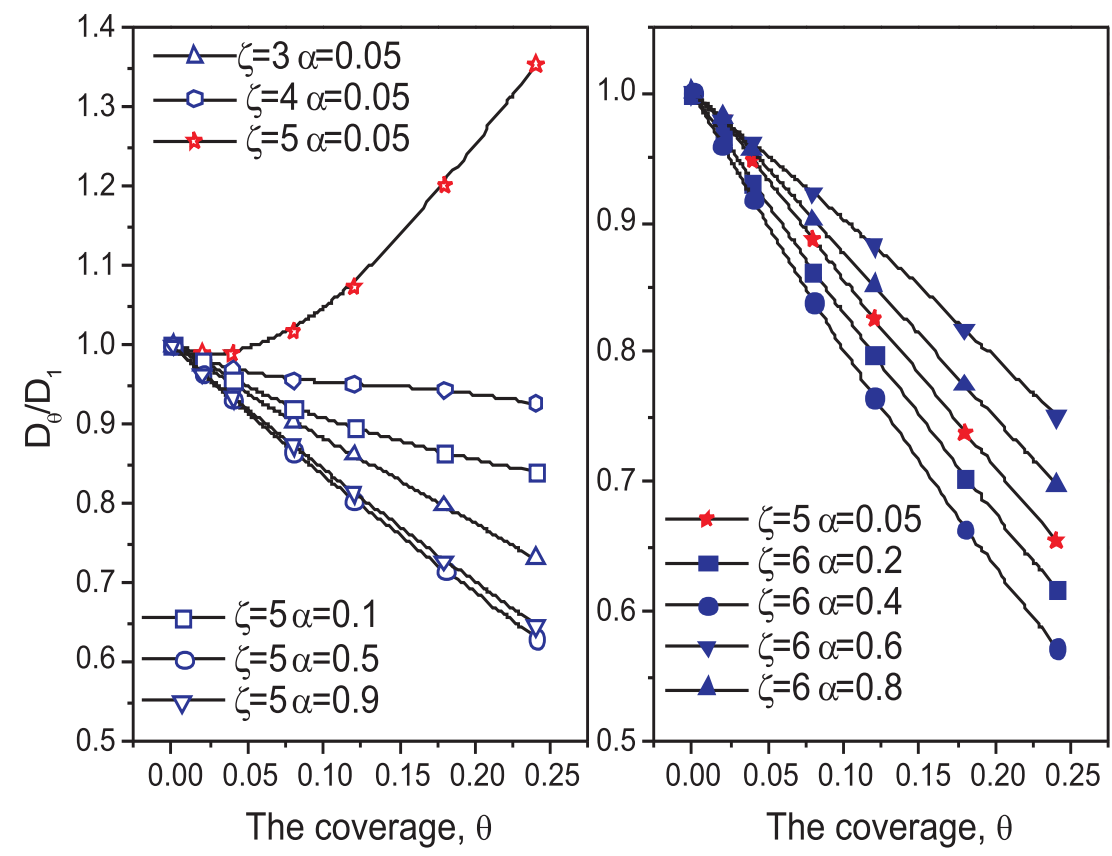

Fig. 6. The coverage dependence of coeffiction diffusion D: lattice A (left) and lattice B (right).

The correlation factor $F$ and time $\tau_{\text {jump }}$ for the lattice with many particles are shown in figures 7 and 8 . For both lattices the factor $F$ significantly decreases with increasing the coverage $\theta$. This is caused by that as the coverage increases, the number of unsuccessful jumps is increased too, e.g. due to the site occupied by another particle some jumps can not be realized and the particle remains in its present site. Regarding to the time $\tau_{\text {jump }}$ one can see two opposite trends: the time $\tau_{j u m p}$ increases for lattice $\mathrm{B}$ and decreases for lattice A (see Fig. 8). In lattice A if all sites with low site energy are occupied, then remaining particles need to move through the site with high energy. As a result, the time $\tau_{\text {jump }}$ decrease. For lattice B the particles prevent other ones to move through the path enriched low transition energies. Consequently, some particles need to surmount high barrier and leads to increase the time $\tau_{\text {jump}}$. As such we observe two opposite trends for time $\tau_{\text {jump }}$. According to Eq. (6) the diffusion coefficient may decrease or increase depending on the ratio between the factor $F$ and time $\tau_{\text {jump }}$. As just mentioned, for lattice A both factor $F$ and time $\tau_{\text {jump }}$ decrease with increasing the coverage, therefore depending on the strength of two these factors the diffusion coefficient may be increased or decreased that observed in the Fig. 6. The dependence of the activation energy and the pre-exponential factor $D_{0}$ on the coverage $\theta$ in Table 2 . It shows that the activation does not affect the coverage, but the pre-exponential factor $D_{0}$ decreases with increasing the coverage $\theta$. 

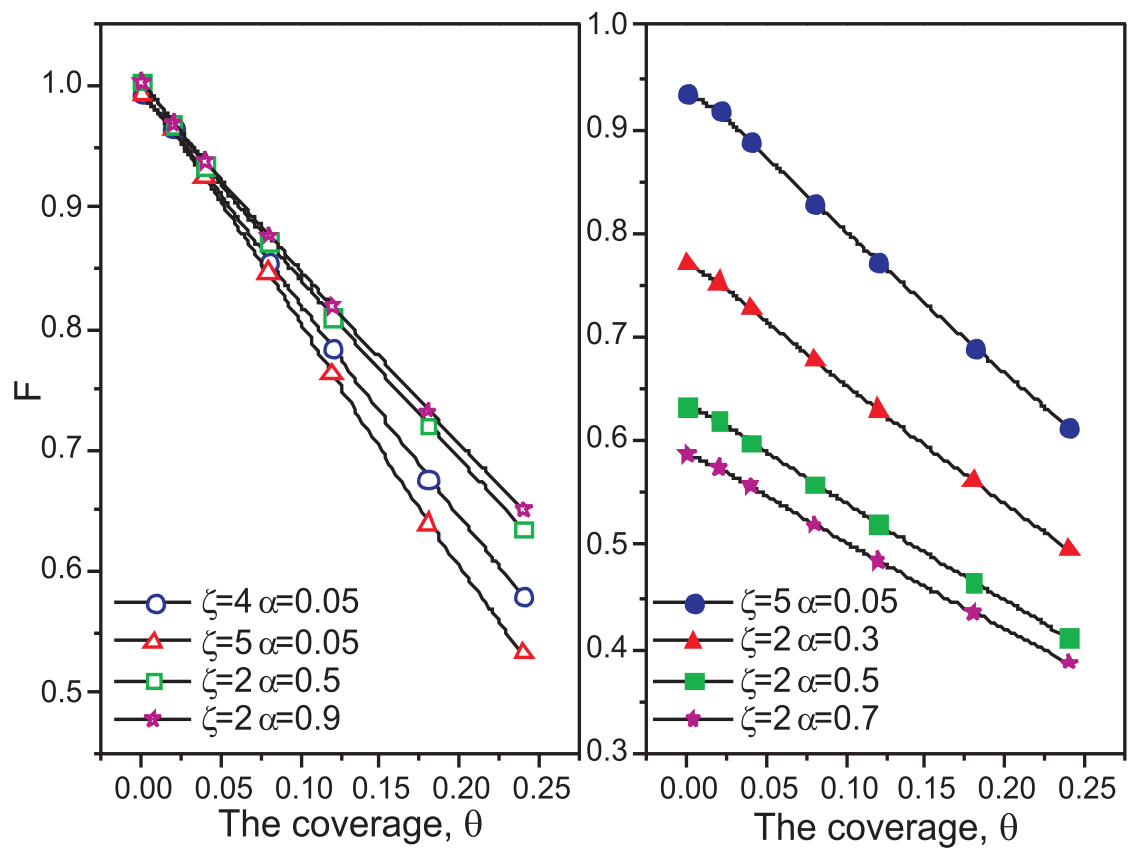

Fig. 7. The coverage dependence of correlation factor F: lattice A (left) and lattice B (right).
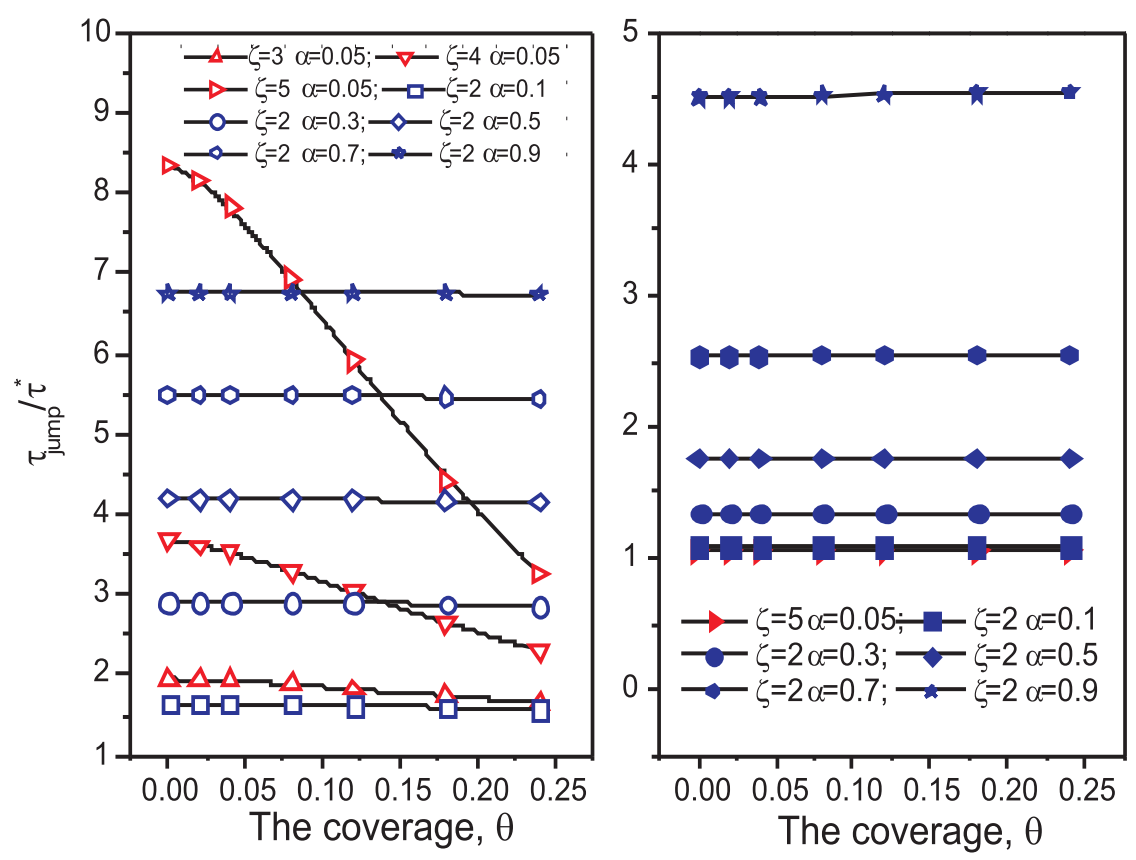

Fig. 8. The coverage dependence of time $\tau_{\text {jump }} / \tau^{*}$ : lattice A (left) and lattice B (right). 
Table 2. The dependence of the activation energy and the pre-exponential factor on the coverage $\theta$

\begin{tabular}{|c|c|c|c|c|c|c|c|c|}
\hline \multirow{3}{*}{$\theta$} & \multicolumn{8}{|c|}{ Lattice A } \\
\hline & \multicolumn{2}{|c|}{$\alpha_{\text {site }}=0.3$} & \multicolumn{2}{|c|}{$\alpha_{\text {site }}=0.5$} & \multicolumn{2}{|c|}{$\alpha_{\text {site }}=0.7$} & \multicolumn{2}{|c|}{$\alpha_{\text {site }}=0.9$} \\
\hline & $\mathrm{E}$ & $\mathrm{D}_{0}$ & $\mathrm{E}$ & $\mathrm{D}_{0}$ & $\mathrm{E}$ & $\mathrm{D}_{0}$ & $\mathrm{E}$ & $\mathrm{D}_{0}$ \\
\hline 0.0004 & 0.94 & 2.34 & 0.97 & 1.72 & 0.99 & 1.33 & 1.00 & 1.10 \\
\hline 0.02 & 0.94 & 2.30 & 0.97 & 1.66 & 0.99 & 1.29 & 1.00 & 1.06 \\
\hline 0.04 & 0.94 & 2.22 & 0.97 & 1.60 & 0.99 & 1.25 & 1.00 & 1.02 \\
\hline 0.08 & 0.94 & 2.07 & 0.97 & 1.49 & 0.99 & 1.17 & 1.00 & 0.96 \\
\hline 0.12 & 0.94 & 1.93 & 0.97 & 1.39 & 0.99 & 1.09 & 1.00 & 0.89 \\
\hline 0.18 & 0.94 & 1.72 & 0.97 & 1.24 & 0.99 & 0.97 & 1.00 & 0.80 \\
\hline 0.24 & 0.94 & 1.53 & 0.97 & 1.10 & 0.99 & 0.86 & 1.00 & 0.71 \\
\hline \multirow{3}{*}{$\theta$} & \multicolumn{8}{|c|}{ Lattice B } \\
\hline & \multicolumn{2}{|c|}{$\alpha_{\text {tran }}=0.3$} & \multicolumn{2}{|c|}{$\alpha_{\text {tran }}=0.5$} & \multicolumn{2}{|c|}{$\alpha_{\text {tran }}=0.7$} & \multicolumn{2}{|c|}{$\alpha_{\text {tran }}=0.9$} \\
\hline & $\mathrm{E}$ & $\mathrm{D}_{0}$ & $\mathrm{E}$ & $\mathrm{D}_{0}$ & $\mathrm{E}$ & $\mathrm{D}_{0}$ & $\mathrm{E}$ & $\mathrm{D}_{0}$ \\
\hline 0.0004 & 0.10 & 0.66 & 0.49 & 0.95 & 0.89 & 1.45 & 0.98 & 1.15 \\
\hline 0.02 & 0.10 & 0.65 & 0.49 & 0.93 & 0.89 & 1.41 & 0.98 & 1.12 \\
\hline 0.04 & 0.10 & 0.63 & 0.49 & 0.90 & 0.88 & 1.36 & 0.98 & 1.08 \\
\hline 0.08 & 0.10 & 0.59 & 0.49 & 0.84 & 0.88 & 1.25 & 0.98 & 1.01 \\
\hline 0.12 & 0.10 & 0.55 & 0.49 & 0.78 & 0.87 & 1.15 & 0.98 & 0.94 \\
\hline 0.18 & 0.10 & 0.49 & 0.49 & 0.69 & 0.87 & 1.01 & 0.98 & 0.84 \\
\hline 0.24 & 0.10 & 0.44 & 0.49 & 0.61 & 0.86 & 0.89 & 0.98 & 0.75 \\
\hline
\end{tabular}

\section{CONCLUSION}

MC simulation is carried out to study the diffusion in the disordered lattices with two types of energetic disorders. Several remarks can be done as

(i) In the case of one particle the diffusion behavior for the lattices with transition and site disorder is quite different: The correlation factor $F$ for the lattice A is monotony decreased with decreasing temperature; meanwhile it is independent of temperature for the lattice B. For two-level distribution of transition energies the factor $F$ attains a minimum near the concentration $\alpha=0.75$ which is independent of the temperature. By analogy the temperature function of time $\tau_{j u m p}$ is quite different for both lattices: for lattice $\mathrm{A}$ the time $\tau_{\text {jump }}$ slightly changes with temperature, whereas it exponentially increases for lattice B. At regime of high concentration $\alpha$ the diffusion follows Arrhenius law for both lattices, but at the interval of $\alpha$ from 0.1 to 0.4 the deviation from Arrhenius behavior is clearly observed.

(ii) The blocking effect in most cases considered depends weakly on the temperature. The ratio $D_{\theta} / D_{1}$ changes from 1.0 to 0.65 and 1.35 to 0.68 for the lattice $\mathrm{B}$ and A respectively. For lattice $\mathrm{B}$ the ratio $D_{\theta} / D_{1}$ decreases in likely-linear manner with increasing the coverage. In the case of lattice $\mathrm{A}$ an anomalous behavior of $D_{\theta} / D_{1}$ is observed near $\alpha=0.05$ and at low temperature. This behavior disappears as the temperature or 
concentration $\alpha$ increases. The origin consists in that both factor $F$ and time $\tau_{j u m p}$ decreases with increasing the particle coverage. As a result of these two opposite factors the $D_{\theta} / D_{1}$ increases upon $\alpha=0.05$ and $\zeta>5.0$.

\section{REFERENCES}

[1] J. W. Haus and K. W. Kehr, Phys. Rep. 150 (1987) 263

[2] Peter M.Richards, Phys. Rev. B16 (4) (1977) 1393

[3] Li-Shi Luo et al., Phys. Rev. E51 (1) (1995) 43

[4] A. V. Nenashev, F. Jansson, S. D. Baranovskii, R. Österbacka, A. V. Dvurechenskii, and F. Gebhard, Phys. Rev. B81 (2010) 115203

[5] J. W. Van de Leur and A. Yu. Orlov, Phys. Lett. A373 (31) (2009) 2675

[6] Y. Limoge and J. L.Bocquet, J. Non-cryst. Solids 117/118 (1990) 605

[7] Y.Limoge and J. L. Bocquet, Phys. Rev. Lett. 65 (1) (1990) 60

[8] Panos Argyrakis et al., Phys. Rev. E52 (4) (1995) 3623

[9] A. Tarasenko and L. Jastrabik, Applied Surface Science 256 (2010) 5137

[10] Tarasenko and L. Jastrabik, Surface Science 602 (2008) 2975

[11] R. Kirchheim and U. Stolz, Acra Metall. 35 (2) (1987) 281

[12] Laurens D. A, Siebbeles, and Yuri A.Berlin, Chem. Phys. Lett. (1997) 460

[13] P. K. Hung, T. V. Mung, L.T. Vinh, Phys. Lett. A373 (2009) 2077

[14] P. K. Hung and D. K. Belashchenco, Izv.Akad.Nauk. SSSR, Metally 2 (1986) 57

[15] P. Argyrakis, E. Arapaki, S.V. Dubinin, Y.G. Groda, and V.S. Vikhrenko, Solid State Ionics 179 (2008) 143

[16] Henk van Beijeren, K. W. Kehr and R. Kutner, Phys. Rev. B28 (10) (1983) 5711

[17] S. H. Payne and H. J. Kreuzer, Phys. Rev. B75 (2007)115403

[18] T. Apih, M. Bobnar, J. Dolinsěk, L. Jastrow, D. Zander, U. Kö̈ter, Solid State Commun. 134 (2005) 337

[19] N. Eliaz, D. Fuks, and D. Eliezer, Mater. Lett. 39 (1999) 255

[20] V. V. Kondratyev, A. V. Gapontsev, A. N. Voloshinskii, A. G. Obukhov, and N. I. Timofeyev, Inter. J. of Hydrogen Energy 13 (1999) 708

[21] Y.-S. Su and S. T. Pantelides, Phys. Rev. Lett. 88 (16) (2002) 165503

[22] G. Majer, U. Eberle, F. Kimmerle, E. Stanik, S. Orimo, Physica B328 (2003) 81

[23] S. J. Manzi, G. A. Ranzuglia, and V. D. Pereyra, Phys. Rev. E80 (2009) 062104

Received 07 May 2011. 